\title{
Dental sleep medicine training moves online
}

Dental sleep medicine (DSM) courses and training are to go online, provided by the British Academy of Dental Sleep Medicine (BADSM) in affiliation with the British Society of Dental Sleep Medicine (BSDSM).

BSDSM is a not-for-profit organisation for dental and medical professionals with an interest in sleep medicine as a subspecialty. To further advance education in DSM, Co-founder and President, Dr Aditi Desai set up the BADSM with Dr Mayoor Patel, Specialist in Sleep and Orofacial Pain from the USA.

The recent broadening of the definition of DSM includes sleep-related breathing disorders (snoring, obstructive sleep apnoea); orofacial pain (OFP); oral moistening disorders (oral dryness, hypersalivation); gastro-oesophageal reflux disorder (GERD); and mandibular movement disorders (dyskinesia, dystonia, sleep bruxism), ${ }^{1}$ which further broadens the scope of practice. Membership of BSDSM and BADSM encourages a multidisciplinary approach to optimise best management outcome for patients with sleep disordered breathing and other conditions. ${ }^{1}$

The Board of BSDSM, led by Dr Desai, comprises medical and dental sleep medicine experts developing and updating guidelines and protocols to promote not only safe practice, but best patient experience.

The Society is responsible for the regulatory aspects of practising DSM with guidance on indemnity and practice protocols. It keeps members up to date with the latest scientific literature and encourages continuing education by enrolling on courses with the Academy, the educational platform for DSM.

Contact the organisations for further information by visiting www.bsdsm.org.uk and www.badsm.org.uk.

\section{Reference}

1. Lobbezoo, F, Aarab G, Wetselaar P, Hoekema A, de Lange J, de Vries N. A new definition of dental sleep medicine. J Dent Sleep Med 2018; 5: 109-112.

\section{Dedicated PPE website launched}

As a global leader in the manufacture of PPE, $3 \mathrm{M}$ Oral Care has now launched a dedicated website providing more information on the products you need to keep safe.

Solutions available for dental professionals include:

- 3M Aura Particulate Health Respirator FFP2, Type IIR 1862+

- $3 \mathrm{M}$ Aura Disposable Health Respirator

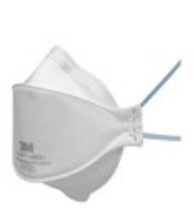

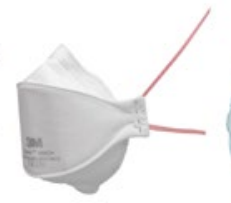
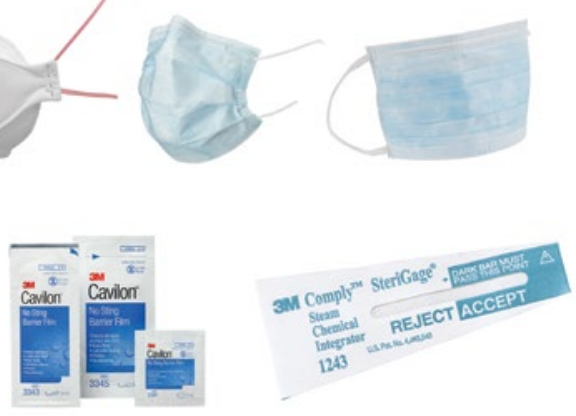

FFP3, Type IIR, 1863+

- Surgical Mask 1826

- Splash Resistant Surgical Mask 1820S

- 3M Triple Layer Molded face Mask 2042F

- $3 \mathrm{M}$ Cavilon No Sting Barrier Film

- 3M Comply SteriGage Integrating Indicator.

Useful information and details of each product can be found on the easy-to-navigate website. Visit 3M.co.uk/dental-ppe today to find out more.

For more information, or to request a video call with your local representative, call 08705360036 or visit 3M.co. uk/dental-ppe.

3M, Aura, Cavilon Comply and SteriGage are trademarks of the 3M Company.

\section{Easy oral hygiene}

Who knew oral hygiene could be so simple with the Hydrosonic Easy toothbrush from Curaprox? This innovative device is compatible with all Curaprox brush heads, but comes complete with the CHS 200 and CHS 300 as standard.

While the CHS 200 is suited to beginners, the CHS 300 is ideal for experienced electric toothbrush users. Both brush heads feature ultra-soft CUREN filaments that are gentle on teeth and gums, but ensure effective removal of plaque - even in hard-to-reach areas.

When it comes to helping patients optimise oral hygiene, it's as easy as recommending the Hydrosonic Easy toothbrush from Curaprox.

For more information call 01480 862084, email info@curaprox.co.uk or visit www.curaprox.co.uk.

\section{Help navigating the labyrinth}

With so many insurance policies to choose between, including vital policies that you need to protect your practice, income and investments, it's easy to see why insurance can be a difficult labyrinth to navigate.

So why not get expert advice from those who can lead you through the maze towards solutions that benefit you best?
At insurance4dentists, the team always make it their goal to offer trustworthy, impartial advice about insurance that will leave you protected and paying the best possible price for your needs. Their specialist knowledge can help you to review your current policies and ensure strong protection for your business in the wake of the COVID-19 pandemic.
As the team only collaborate with dental professionals, they have a unique understanding of the current and future needs of dentists, and use this knowledge to find insurance policies that are best tailored to your every need.

For more information call 0845345 5060, email info@money4dentists.com or visit www.money4dentists.com. 